\title{
Everyday Living Artificial Intelligence Hub
}

\author{
Raymond Finzel $^{1}$, Esha Singh ${ }^{2}$, Martin Michalowski ${ }^{3}$, Serguei Pakhomov $^{1}$, Maria Gini ${ }^{2}$ \\ ${ }^{1}$ Department of Pharmaceutical Care and Health Systems, College of Pharmacy, \\ ${ }^{2}$ CSE Department, College of Science and Engineering, \\ ${ }^{3}$ Population Health and Systems Cooperative, School of Nursing, \\ University of Minnesota, Twin Cities, Minnesota, United States of America. \\ \{finze006, sing0640, martinm, pakh0002,gini\}@umn.edu
}

\begin{abstract}
We present the Everyday Living Artificial Intelligence (AI) Hub, a novel proof-of-concept framework for enhancing human health and wellbeing via a combination of tailored wearable and Conversational Agent (CA) solutions for non-invasive monitoring of physiological signals, assessment of behaviors through unobtrusive wearable devices, and the provision of personalized interventions to reduce stress and anxiety. We utilize recent advancements and industry standards in Internet of Things (IoT) and AI technologies to develop this proof-ofconcept framework.
\end{abstract}

\section{Introduction}

The significance of stress in disease development and progression has been established for multiple therapeutic areas including cardiovascular disease (Kivimäki and Steptoe, 2018), type 2 diabetes (Hackett and Steptoe, 2017), obesity (Sinha and Jastreboff, 2013), sleep disorders (Han et al., 2012), depression (Madsen et al., 2017), stroke (O'Donnell et al., 2016) drug addiction (including opioid, tobacco, cannabis, and cocaine use) (Airagnes et al., 2018; Preston et al., 2017) , and Alzheimer's disease (Justice, 2018). As demonstrated for cardiovascular disease, stressors associated with increased risk of events include those commonly encountered in life such as work stressors, anger episodes and even the viewing of stressful sporting events (Smyth et al., 2016). Exposure to stressful events is therefore a major risk factor for morbidity and mortality rates especially for conditions that have a great impact on public health.

As the commercial IoT sector continues to grow, our homes and bodies are increasingly instrumented. We now have digital personal assistants that listen and respond to voice commands and wearable devices equipped with multiple sensors. The availability and maturity of this technology affords an unprecedented opportunity to develop holistic systems to advance the health and wellbeing of many groups including one of the most vulnerable sectors of our population: elders aging in-place. To provide personalized and effective interventions, such a system must be capable of sensing, integrating, responding to physical, emotional, and cognitive status in an accessible way.

Towards this vision, we have developed a proofof-concept framework for individually tailored detection and management of mental stress and anxiety in everyday life. We target the development and deployment of a novel personalized technology that integrates conversational voice assistants with wearable sensors and smart-textile clothing technology to provide real-time, in-home, unobtrusive sensing and on-body stimulation solutions (e.g., pressure, heat, etc.). The proposed proofof-concept framework integrates three major components: 1) natural language interaction with the user via a conversational voice assistant; 2 ) physiological signal sensing of activity, heart rate, body temperature, and electrical conductivity of skin; and 3) garment-based delivery of heat and compression interventions to reduce stress and anxiety detected via voice and wearable sensors.

\section{Methodology}

The Everyday Living AI Hub is a holistic framework that orchestrates the 3 components discussed in section 1, coordinating multiple streams of biometric data and physical interventions (Figure 1). The Hub framework allows for the analysis of selfsupplied information (such as information about a user's schedule, habits, and preferences) alongside biometric data collected from OTC wearable devices with the goal of providing interventions into the user's life to help them self-regulate. These interventions are in the form of notifications (reminders to breathe, reminders that a meeting or other scheduled time is coming up), or bindings with devices that operate "In Real Life (IRL)" such 


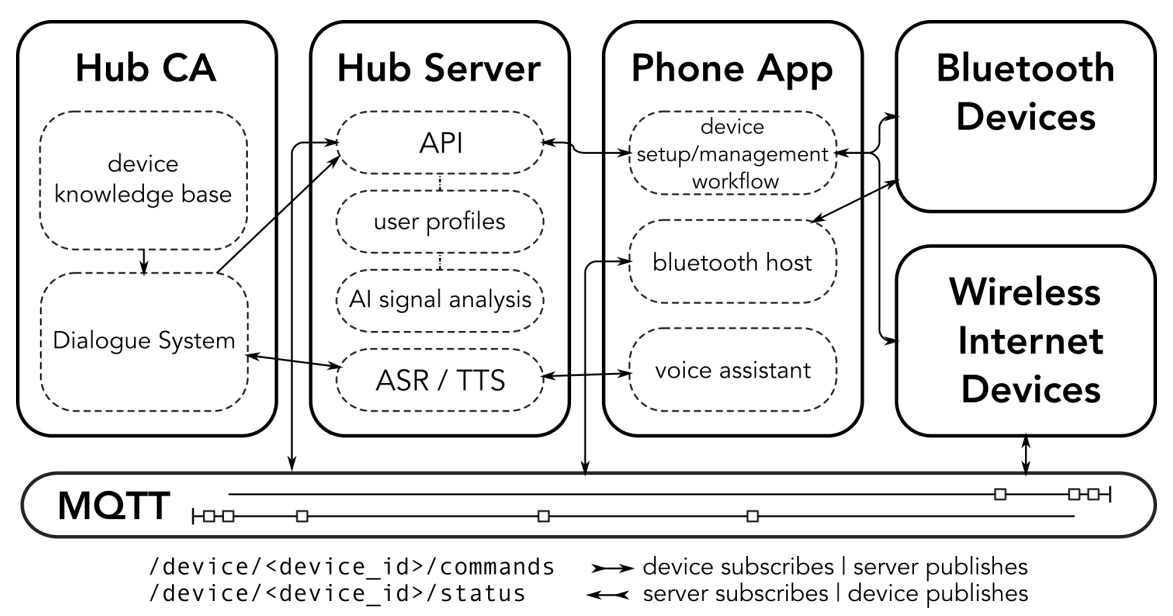

Figure 1: Architecture for Everyday Living AI Hub and end-point user interaction flow.

as the SmartHugs Garment (Pettys-Baker et al., 2018), a wearable shirt designed to perform compression on dysregulated individuals. Detected stress events are confirmed with the user via the Hub Conversational Agent (Hub CA), which can ask questions about the severity and timing of the stressful event, and confirm whether an intervention is necessary or desirable.

Earlier stress measures were mainly questionnaires such as Demand-Control-Support model (Johnson and Hall, 1988, Pozo-Antúnez et al., 2018, Karasek and Theorell, 1990). Lu et al. proposed a framework for real-time stress measurement, monitoring and intervention. Like our proposed system, they used physiological indicators to detect stress level using a wearable smart bracelet. Our contribution adds human-in-the-loop voice control and the ability to incorporate interventions via IoT devices to the milieu of this prior work.

\subsection{Architecture}

By utilizing many industry-standard IoT protocols, the framework is designed to be flexible. User accounts and profiles are maintained via a web service based on Responder (Reitz, 2018), a free Python framework for microservice development. Monitoring devices collect data and send it through Bluetooth to a cell-phone companion app, or send it directly to the Hub Server's AI collection point via a lightweight publish/subscribe protocol called MQTT (Figure 1). Everyday interactions with the framework are performed either by interacting with a web-service Application Programming Interface (API) via mobile application, website, or by using natural language to converse with the voice assistant, which passes speech data through an Au- tomatic Speech Recognition (ASR) service to the Hub CA which is based on MindMeld (Raghuvanshi et al., 2018), a framework for conversational agent development. The Hub CA then performs actions on behalf of the user by interacting with the API, and speaks to the user using the integrated Text-To-Speech (TTS) service.

\subsection{Project Status}

Completed modules include system architecture components such as MQTT services, web APIs for data collection and intervention applications, a phone app for the management of Bluetooth connected devices, ASR and TTS modules, and a prototype stress management garment. Active development is underway for AI signal analysis, in-theloop conversational device setup, and intervention management in the Hub CA.

\section{Challenges}

The Everyday Living AI Hub requires many practical and theoretical advances. While preliminary studies have shown that commercial IoT wearables can detect changes in heart-rate that correspond to stress in a naturalistic environment (Pakhomov et al., 2020), existing machine-learning models are not equipped to reliably predict stressful events in real-time. Collection of data and the creation of an adequate model are ongoing work. The Hub CA is based on established chatbot paradigms, but models and dialog flows are still under development for the new domain of stress management.

\section{Acknowledgements}

UMN Grand Challenges Research Initiative. 


\section{References}

Guillaume Airagnes, Cédric Lemogne, Marcel Goldberg, Nicolas Hoertel, Yves Roquelaure, Frédéric Limosin, and Marie Zins. 2018. Job exposure to the public in relation with alcohol, tobacco and cannabis use: Findings from the constances cohort study. PLOS ONE, 13:e0196330.

Ruth Hackett and Andrew Steptoe. 2017. Type 2 diabetes mellitus and psychological stress - a modifiable risk factor. Nature reviews. Endocrinology, 13.

Kuem Han, Lin Kim, and Insop Shim. 2012. Stress and sleep disorder. Experimental neurobiology, 21:14150 .

Jeffrey Johnson and E.M. Hall. 1988. Job strain, work place social support, and cardiovascular disease: A cross-sectional study of a random sample of the swedish working population. American journal of public health, 78:1336-42.

Nicholas J. Justice. 2018. The relationship between stress and alzheimer's disease. Neurobiology of Stress, 8:127-133.

Robert Karasek and Töres Theorell. 1990. Healthy Work: Stress, Productivity, and The Reconstruction Of Working Life.

M. Kivimäki and A. Steptoe. 2018. Effects of stress on the development and progression of cardiovascular disease. Nature Reviews Cardiology, 15:215-229.

Peixian Lu, Wei Zhang, Liang Ma, and Qichao Zhao. 2020. A Framework of Real-Time Stress Monitoring and Intervention System, pages 166-175.

Ida E. H. Madsen, S. Nyberg, Linda Magnusson Hanson, Jane Ferrie, Kirsi Ahola, Lars Alfredsson, G. Batty, Jakob Bjorner, Marianne Borritz, Hermann Burr, J.-F Chastang, Ron Graaf, Nico Dragano, Mark Hamer, M. Jokela, Anders Knutsson, M. Koskenvuo, Aki Koskinen, Constanze Leineweber, and Minna Kivimäki. 2017. Job strain as a risk factor for clinical depression: systematic review and meta-analysis with additional individual participant data. Psychological Medicine, 47:1-15.

Martin O'Donnell, Siu Chin, Sumathy Rangarajan, Denis Xavier, Lixin Liu, Hongye Zhang, Purnima RaoMelacini, Xiaohe Zhang, Prem Pais, Steven Agapay, Patricio Lopez-Jaramillo, Albertino Damasceno, Peter Langhorne, Matthew McQueen, Annika Rosengren, Mahshid Dehghan, Graeme Hankey, Antonio Dans, Ahmed Elsayed, and Yan Duarte. 2016. Global and regional effects of potentially modifiable risk factors associated with acute stroke in 32 countries (interstroke): A case-control study. The Lancet, 388 .

Serguei Pakhomov, Paul Thuras, Raymond Finzel, Jerika Eppel, and Michael Kotlyar. 2020. Using consumer-wearable technology for remote assessment of physiological response to stress in the naturalistic environment. PLOS ONE, 15:e0229942.
Robert Pettys-Baker, Nicholas Schleif, J. Walter Lee, Sophia Utset-Ward, Mary Ellen Berglund, Lucy E. Dunne, Brad Holschuh, Christopher Johnson, Kevin Kelly, Bruce Johnson, and Michael Joyner. 2018. Tension-Controlled Active Compression Garment for Treatment of Orthostatic Intolerance. 2018 Design of Medical Devices Conference. V001T10A005.

José Pozo-Antúnez, Antonio Ariza-Montes, Francisco Fernández-Navarro, and Horacio Molina. 2018. Effect of a job demand-control-social support model on accounting professionals' health perception. International Journal of Environmental Research and Public Health, 15.

Jonathan Preston, Megan Leece, Kerry McNamara, and Edwin Maas. 2017. Variable practice to enhance speech learning in ultrasound biofeedback treatment for childhood apraxia of speech: A single case experimental study. American Journal of SpeechLanguage Pathology, 26:1.

Arushi Raghuvanshi, Lucien Carroll, and Karthik Raghunathan. 2018. Developing production-level conversational interfaces with shallow semantic parsing. In Proceedings of the 2018 Conference on Empirical Methods in Natural Language Processing: System Demonstrations, pages 157-162.

Kenneth Reitz. 2018. A familiar http service framework.

R. Sinha and A. Jastreboff. 2013. Stress as a common risk factor for obesity and addiction. Biological Psychiatry, 73:827-835.

Elizabeth Smyth, Matteo Fassan, David Cunningham, William Allum, Alicia Okines, Andrea Lampis, Jens Hahne, Massimo Rugge, Clare Peckitt, Matthew Nankivell, Ruth Langley, Michele Ghidini, Chiara Braconi, Andrew Wotherspoon, Heike Grabsch, and Nicola Valeri. 2016. Effect of pathologic tumor response and nodal status on survival in the medical research council adjuvant gastric infusional chemotherapy trial. Journal of Clinical Oncology, 34. 